\title{
Conservation of selection on matK following an ancient loss of its flanking intron
}

\author{
Aaron M. Duffy ${ }^{a, *}$, Scot A. Kelchner ${ }^{b}$, Paul G. Wolf ${ }^{a}$ \\ a Department of Biology, Utah State University, Logan, UT 84322, USA \\ b Department of Biological Sciences, Idaho State University, Pocatello, ID 83209, USA
}

\section{A R T I C L E I N F O}

\section{Article history:}

Received 10 October 2008

Received in revised form 29 January 2009

Accepted 10 February 2009

Available online 21 February 2009

Received by G. Theissen

\section{Keywords:}

Group II intron

Secondary structure

$\operatorname{trnK}$

Intron-encoded protein

Fern

\begin{abstract}
A B S T R A C T
The chloroplast gene trnK and its associated group II intron appear to be absent in a large and ancient clade that includes nearly $90 \%$ of fern species. However, the maturase protein encoded within the intron (matK) is still present and located on the boundary of a large-scale inversion. We surveyed the chloroplast genome sequence of clade-member Adiantum capillus-veneris for evidence of a still present but fragmented trnK intron. Lack of signature structural domains and sequence motifs in the genome indicate loss of the trnK intron through degradation in an ancestor of the clade. In plants, matK preferentially catalyzes splicing of the trnK intron, but may also have a generalist function, splicing other group II introns in the chloroplast genome. We therefore tested whether a shift in selective constraint has occurred after loss of the trnK intron. Using previously unavailable sequences for several ferns, we compared matK sequences of the intron-less fern clade to sequences from seed plants and ferns with the intron and found no significant differences in selection among lineages using multiple methods. We conclude that matK in ferns has maintained its apparently ancient and generalized function in chloroplasts, even after the loss of its co-evolved group II intron. Finally, we also present primers that will allow amplification and nucleotide sequencing of the phylogenetically useful matK gene in additional fern taxa.
\end{abstract}

(c) 2009 Elsevier B.V. All rights reserved.

\section{Introduction}

The plant chloroplast gene matK has long sparked the interest of molecular evolutionary biologists. Its open reading frame is associated with a group II intron (Fig. 1A) that interrupts the coding sequence of tRNA $^{\text {Lys }}$ (UUU) and it shows a much faster rate of sequence evolution than many other chloroplast genes (Wolfe et al., 1992; Hilu and Liang, 1997). The discovery of matK's presence in the highly reduced plastomes of nonphotosynthetic plants (Wolfe et al., 1992; Ems et al., 1995) surprised many researchers and pointed to its probable role as a maturase that catalyzes the splicing reactions of more than one group II intron in the chloroplast genome (e.g., Ems et al., 1995; Liere and Link, 1995; Vogel et al., 1999).

Many known group II introns possess their own intron-encoded protein (IEP), which assists in splicing its host intron (Toor et al., 2001; Hausner et al., 2006). However, nearly all of the 20 or so group II introns in plant plastomes show severe degradation of their maturase open reading frames. This condition suggests that plant chloroplast group II introns no longer need to maintain their own splicing cofactor, an observation that many consider to be a strong indication of

Abbreviations: IEP, intron-encoded protein; ORF, open reading frame; CTAB, Cetyl trimethylammonium bromide; nt, nucleotide; RT, reverse-transcriptase; mya, million years ago.

* Corresponding author. Tel.: +1 435797 0052; fax: +1 4357971575 .

E-mail address: aduffy@biology.usu.edu (A.M. Duffy).
matK's role as a generalist maturase (reviewed by Hausner et al., 2006).

The purported generalist function of matK would be unusual for an intron maturase. Introns and their IEPs are thought to have coevolved: similar phylogenetic relationships are found among IEP sequences as are found among their intron RNA structures (Toor et al., 2001 ). Hence, there is a strong likelihood that the two components of a complete intron sequence (the intron itself, and its IEP open reading frame) are indelibly linked in terms of structure and function. Any shift in the intron's primary sequence or secondary and tertiary structure would likely correlate with a change of its IEP sequence (and function) if it is to successfully pass through the filters of natural selection.

Interestingly, the catalysis link between host intron and IEP continues to be strong in matK, even though it may also function as a generalist splicing co-factor for many chloroplast introns. Vogel et al. $(1997,1999)$ have shown in vivo that matK is required for trnK intron splicing in barley, and it will preferentially catalyze this reaction over the presumably less specific splicing of additional chloroplast introns, particularly those of structural subclass IIA (Liere and Link, 1995).

The unusual role of matK led us to question how selective constraints might vary for matK sequences in cases where the gene is no longer associated with its principal target, the trnK intron. This condition was observed in the chloroplast genome of the fern Adiantum capillus-veneris, which possesses matK and shows evidence of matK transcription (Wolf et al., 2004), yet appears to lack trnK and 
A

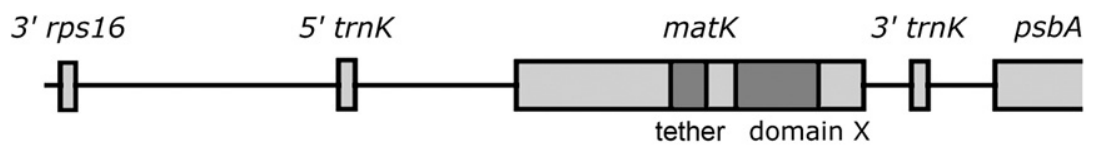

$1234 \quad 56$

trnK intron

B

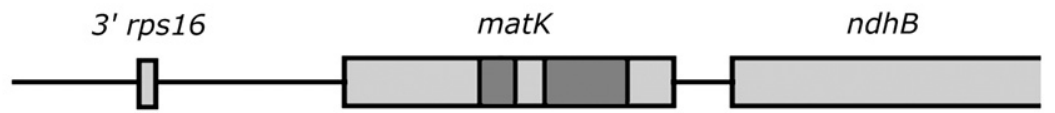

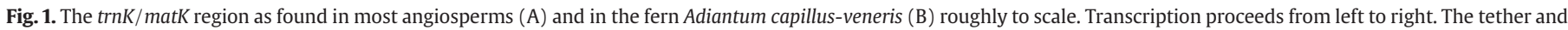
domain $\mathrm{X}$ are relatively conserved functional regions of the matK protein. Numbers 1 through 6 designate the location of domains 1 through 6 of the trnK intron, respectively.

its intron (Wolf et al., 2003; Fig. 1B). Genome mapping studies indicate that the loss of trnK and its intron is associated with an ancient inversion event in the ancestor of a large clade of leptosporangiate ferns (Hasebe and Iwatsuki, 1992, Stein et al., 1992; Roper, 2007). This is an old lineage ( 265 mya), which includes nearly $90 \%$ of the approximately 11000 extant fern species (Pryer et al., 2004). This genome structure would also explain the failure to obtain matK sequence from ferns: the PCR primers used in other plants are located in the missing trnK exons (e.g. Hilu and Liang, 1997; Hilu et al., 2003; Hausner et al., 2006).

Cases of trnK intron loss with matK retention have been inferred only twice before in plants: once in the highly reduced chloroplast genome of the achlorophyllous parasitic plant Epifagus virginiana (Wolfe et al., 1992; Ems et al., 1995) and once in the chloroplast genome of Cuscuta reflexa, a parasitic plant with reduced photosynthetic activity (Funk et al., 2007). However, these are recent changes that may not be shared with other extant taxa of each lineage, whereas the fern example is likely due to a very old event resulting in an intron-less matK in the majority of extant fern species. Ferns therefore present an opportunity to study the possible shift of selective constraints on an IEP (matK) after isolation from its coevolved intron (the trnK intron) in a well-sampled and ancient clade of plants.

In this study, we use computational methods to establish whether the trnK intron is indeed absent from the A. capillus-veneris chloroplast genome or is instead divided but still functional, by searching for conserved intron sequence elements and intron-specific secondary structures. We then test whether any of matK's major protein domains have experienced a shift in selective constraints after the loss of the trnK intron. We did this by: (1) obtaining matK sequences for additional fern taxa with and without a contiguous trnK intron, (2) comparing patterns of nucleotide and amino acid conservation across matK sequences in ferns and also seed plants, and (3) comparing rates of nonsynonymous to synonymous nucleotide substitutions in these groups using several methods. We also present primers for amplifying and sequencing a portion of the matK gene in ferns that are missing the trnK intron.

\section{Materials and methods}

\subsection{Search for trnK intron in Adiantum}

The trnK intron is not present in its expected location in the A. capillus-veneris chloroplast genome (Wolf et al., 2003), although this observation alone does not confirm its complete absence. Recombination involving group II introns has led to many known cases in which intron fragments are dislocated in a genome yet retain their function through trans-splicing mechanisms (Chapdelaine and Bonen, 1991;
Bonen, 1993; Ems et al., 1995; Knoop et al., 1997; Jarrell et al., 1988; Malek and Knoop, 1998; Qiu and Palmer, 2004). Hence, it is important to our study that we first confirm an absolute loss of a conserved trnK intron sequence in the A. capillus-veneris chloroplast genome prior to interpreting any shift in patterns of selection among matK sequences that might be indicative of a change in the protein's function. We devised a method that first tests for the uniqueness of sequence within highly conserved structures of the trnK intron and then identifies several small sequence elements that would assist in locating intron structural fragments within the A. capillus-veneris chloroplast genome.

We constructed estimated secondary structure models for the trnK intron/matK sequence regions from Pellia borealis, Marchantia polymorpha, Sphagnum platyphyllum, Cycas panzhihuaensis, Pinus thunbergii, and Atropa belladonna (Supplementary Table 1; see Hausner et al., 2006 for additional trnK intron secondary structure models) using the domain-by-domain folding strategy of Kelchner (2002). The resulting RNA structures were then compared to identify conserved structural elements. Sequences of these structural elements (Table 1) were used to search the intergenic spacer regions immediately upstream and downstream of the matK ORF in A. capillus-veneris and all positive matches were explored using localized RNA folding by Mfold (Zuker, 2003) to survey for possible group II intron secondary structures. Sequences from both the upstream and downstream noncoding regions surrounding the $A$. capillus-veneris matK ORF were also folded with Mfold using an arbitrary "sliding window" approach with multiple sequence lengths shifting outward from the matK region in an attempt to identify helices that could be homologous with trnK intron domains. In particular, we looked for evidence of trnK intron domains 1 through 4 , which would likely be proximal upstream of the matK ORF (Fig. 1).

The nucleotide sequence of domain 5 of the trnK intron is relatively well conserved among land plants (Supplementary Fig. 1) and should be recognizable if it is present in the chloroplast genome. The 34 nucleotides of domain 5 from $P$. borealis, M. polymorpha, S. platyphyllum, C. panzhihuaensis, P. thunbergii, and A. belladonna were used individually for BLAST searches against the GenBank database to verify

Table 1

Sequence elements used in searches for trnK intron fragments in A. capillus-veneris.

\begin{tabular}{llllllllll}
\hline $5^{\prime}$ nt & D2 5' & D2 3' & EBS2 & EBS1 & D3 3' & D4 5' & D4 3' & D5 5' & D5 loop \\
\hline GTGCG & GGAT & ATCC & AGCTT & GTTAGAA & ATCGC & ATGTA & ATCGC & GCCG & GAAA \\
& & & & GTTAAAA & ATTGC & ATGCA & ATTGC & & GAGA
\end{tabular}

All matches identified in the A. capillus-veneris chloroplast genome were subjected to RNA folding to search for trnK intron fragments. Elements correspond to highly conserved trnK intron structures in a liverwort (Pellia borealis), a moss (Sphagnum platyphyllum), a conifer (Pinus thunbergii), and a flowering plant (Atropa belladonna). Nomenclature follows Michel et al. (1989). 
that these nucleotides would identify domain 5 in other land plant trnK sequences, against the Angiopteris evecta chloroplast genome sequence to verify that they would identify domain 5 in a fern, and directly against the $A$. capillus-veneris chloroplast genome sequence as an attempt to locate trnK domain 5 in this genome. Each BLAST (blastn) search employed a low complexity filter with an expect threshold of 10 and word size of 11 , and the return limit was set at the maximum 1000 matches from a Eukaryota virtual database.

\subsection{DNA extraction, amplification, and sequencing}

Taxa were chosen to represent most major fern lineages (Supplementary Table 1). Genomic DNA for new sequences was extracted using Qiagen DNEasy kits or a CTAB method (Doyle and Doyle, 1987). Published seed plant matK primers failed to amplify matK in fern taxa, and attempts to design universal fern matK primers using the matK sequences of $A$. capillus-veneris and $A$. evecta were not successful due to the lack of highly conserved regions. Sequences for matK in Osmunda cinnamomea, Marsilea mutica, and Dicksonia antarctica were obtained by amplifying large fragments using primers in flanking genes and primer walking. Using these sequences and the matK sequences of $A$. capillus-veneris and $A$. evecta, two sets of fern matK primers were designed: one set for the more recently derived "modern" ferns and one set for the more basal "early" ferns (Fig. 2). These primers were used to amplify and sequence portions of matK from Lygodium japonicum and Pteridium aquilinum. Because these primers are located within matK, to obtain the complete matK sequences, flanking regions were amplified and sequenced using these primers combined with primers in flanking genes. We also used the published matK sequences of the lycophyte Huperzia lucidula, the seed plants Pinus koraiensis, Amborella trichopoda, Nymphaea alba, Magnolia dealbata, Helianthus annuus, Triticum aestivum, and Cycas pectinata, and the monilophytes Psilotum nudum, and Ophioglossum petiolatum from GenBank (Supplementary Table 1). The matK sequences of Isoetes engelmannii and Equisetum arvense were extracted from their unpublished complete chloroplast genomes (K. Karol, personal communication).

\subsection{Phylogenetic analysis}

Nucleotide sequences were converted to amino acid sequences and aligned using clustalW with manual refinement informed by the nucleotide sequence and following the alignment principles of Kelchner (2000). The alignment was then converted back to nucleotide sequences for subsequent analyses. A single nucleotide gap was opened after nt 149 in Huperzia to shift the reading frame so the rest of the sequence would align. To match known RNA editing in Adiantum (Wolf et al., 2004), ACG (T) was changed to ATG (M) at nt 5, TCA (S) was changed to TTA (L) at nt 497 , TCC (S) was changed to TTC
(F) at nt 1001, and CCG (P) was changed to CTG (L) at nt 1004. RNA editing, including editing to repair stop codons, appears to be common in the monilophytes (Wolf et al., 2004) and matK in general (Barthet and Hilu, 2007). Several of our sequences contain stop codons that we changed to extend the open reading frame: TAA was changed to CAA (Q) at nt 559 in Isoetes, TGA was changed to CGA (R) and TAA was changed to CAA (Q) at nt 256 and 262 of Dicksonia and at nt 262 and 268 of Pteridium, and TGA was changed to CGA (R) at nt 250 and 256 of Marsilea. Making these changes extended the ORFs to approximately the same length as other matK sequences. Sections of the alignment containing indels that could not be aligned unambiguously were not included in subsequent analyses (Fig. 3).

We performed Bayesian Metropolis coupled Markov chain Monte Carlo analyses using MrBayes v3.1.2 (Huelsenbeck and Ronquist, 2001; Ronquist and Huelsenbeck, 2003). The data were partitioned by codon position and each partition was assigned its own model of nucleotide substitution (GTR + I + gamma) as determined using MrModeltest 2.2 (Nylander, 2004), a modified version of Modeltest 3.6 (Posada and Crandall, 1998). Using a random starting tree, we performed three separate runs with four chains each for 1250000 generations, sampling every 1000 generations. We plotted the log probability of observing the data by generation to detect stationarity and discarded the first 250 samples as "burnin." We pooled the post-burnin trees from each run and calculated a majority-rule consensus tree.

\subsection{Tests of relaxed selective constraint}

We compared the patterns of nucleotide and amino acid conservation along the length of the matK sequence in ferns with the trnK intron, ferns without the trnK intron, and seed plants. By focusing on the pattern of conservation rather than on absolute levels of conservation, we reduce bias due to different sample sizes, relationships between the groups, and whether taxa are equivalent between groups.

Several methods are available for detecting shifts in selection using the ratio of nonsynonymous to synonymous substitution rates ( $\mathrm{d} N /$ $\mathrm{d} S$; reviewed by Yang and Bielawski, 2000). If matK has been under relaxed constraint in the chloroplast genomes of ferns since the loss of trnK and its intron, we should be able to detect an increase in $\mathrm{dN} / \mathrm{d} S$ ratios relative to ferns that have retained trnK and its intron. Those differences might be expected to be most evident in the active regions of the gene: domain $\mathrm{X}$, which is associated with splicing activity, and the "tether region," which represents part of a reverse-transcriptase (RT) domain (Mohr et al., 1993; Hausner et al., 2006; Fig. 1). We tested for shifts in selection specifically on these domains, as well as on the gene as a whole. However, when using these much smaller subsets of the data, no differences in the pattern of substitution rates among the lineages were found and no statistical tests found significance that was not also present for the entire matK sequence, so only the results for the entire matK sequence will be presented.

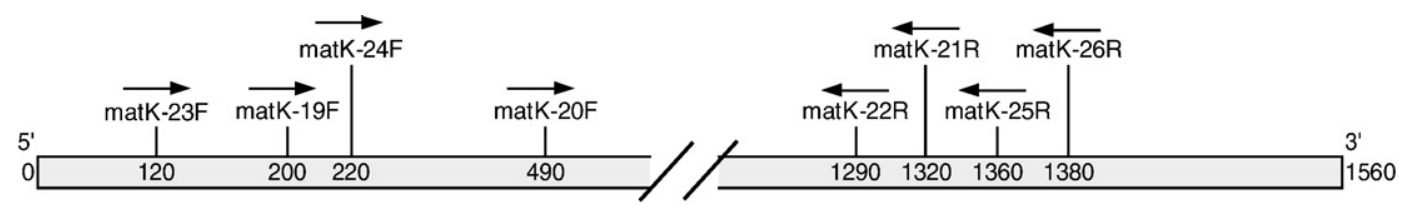

\begin{tabular}{lc}
\hline "modern" fern primers: \\
\hline matK-19F & CGGCGAAACGTTTAATYGRTAG \\
matK-20F & CTTCATTCAGAGACTYYAATTCG \\
matK-21R & ACAGGCTAAMGTAYTATCACATG \\
matK-22R & ATATATTTCAATCTRCGCAAYC \\
\hline
\end{tabular}

\begin{tabular}{ll}
\hline "early" fern primers: \\
\hline matK-23F & AAGATGATTCTTACKCRATTGCT \\
matK-24F & TAATTGATAGGATRCGTCARCAAG \\
matK-25R & CGTGTTGTACTTTTRTGTTTACARGC \\
matK-26R & GTTTACAGGCYAARGTTTTATCGC \\
\hline
\end{tabular}

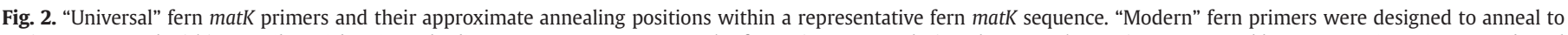

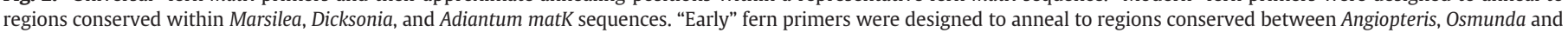
Marsilea. 


\begin{tabular}{|c|c|c|c|c|c|c|c|c|c|}
\hline & $\begin{array}{l}1 \\
1\end{array}$ & $\left.\right|^{11}$ & ${ }_{1}^{21}$ & ${ }^{31}$ & $\begin{array}{l}41 \\
\mid\end{array}$ & $\left.\right|^{51}$ & ${ }_{1}^{61}$ & ${ }^{71}$ & $\left.\right|^{81}$ \\
\hline uperzia & RIDKLQTKRF & LYLLLFQEDI & HAIACNRFSK & KDSSLSFITR & KRLISKIRQQ & NLLEGGGHQS & FDESIREVLT & SVLQIVEEIL & \\
\hline agnolia & MEELQEIQD. & .P.L..Y. & Y.L.HDHGLN & SNFGY.SLIV & . . . TRMH & .H. ISVNDRF & VGQMVS.GFA & VIME.L..KS & \\
\hline diantum & KSGP.GENC. & .HP. . L.NF & YLTDGK.R.H & ALVFG. TVAV & ...GSV.DP & .F.KSVSVDG & L.HPLLKMTY & LI.G.PA.TS & MT \\
\hline teridium & KF.A.EED. & .TF .L.F & YS. .RK. CLD & PLVFG.AVAT & ..S.GG..D. & .YS.SEFVGR & .NHGLL.TIC & LI.E.DVKKS & FS \\
\hline onia & KLGT . KREC. & .PFF.KD.F & YS...K.P.D & PSVFG.M.SA & . . DRM.H. & DYSDSESGS. & SNYALL.TIC & L. .E. SMKRW & \\
\hline ea & KDGGVKKDF. & .T.F.K.S & YS ..YK..PG & SSVFG.I.SS & ..I.DRM . . & DYSRSESGGR & WNYALV.AIC & L.SEKSTKKW & \\
\hline um & STSE.RI.C. & .H.YV..D. & YPT.WTHL.G & LNFHA. I.SV & ........ & .YSKFEYDEN & LGNALLKGIF & IA.E.SL.KW & \\
\hline da & .FE. . RKEC. & AC.S.D.P & Y...Y.C.LN & SN.GF.I.SI & $\ldots$. KG . . . & D. SGFLPDR. & GND. LLKGIA & L..E. E & \\
\hline ceris & K. . . . KRQ . . & .TIP.... & Y...YT.S.. & SN.V..V..I & $\ldots$. TQL . . & .F. KN & YNKLLL.G.I & LI.EVI & \\
\hline um & LFENIV.Q. . & . . P. . HKEF & YVVTSDS.ID & SEFV. . .LVI & R...NR . .NL & IQTK. & ICFYLLKA.N & M. .ETI & \\
\hline ssum & V.GG & S.P...HD.P & .F.R..S.D & SYRNR. ILAV & $\ldots \ldots$ RM.RR & . PY & T.RYL & V..E & \\
\hline \multirow[t]{2}{*}{ Psilotum } & K. G. & .P...R.L & Y...Y..S.S & LHRD & $\ldots \mathrm{N}$ & TTSK & INR. & VLFE & \\
\hline & $\begin{array}{l}111 \\
\mid\end{array}$ & t & $\begin{array}{l}131 \\
\mid\end{array}$ & $\begin{array}{l}141 \\
\mid\end{array}$ & $\begin{array}{l}151 \\
\mid\end{array}$ & ${ }^{16}$ & ${ }_{1}^{171}$ & $\begin{array}{l}181 \\
\mid\end{array}$ & \\
\hline$a$ & FIHP & $A P F$ & SHLLR & YQDK & KLST & VHEF & LWKR & FPL & \\
\hline & P..L & . s & L...L & . RK. & RFFI & .Y.C.V.F & . R. & TS & \\
\hline & $\ldots L$ & W.K & L...I. . RK & RKKT & SVDI & IF.ID.L.LI & P..Q & NY & \\
\hline um & $\ldots L$ & $\ldots Q$ & .......HK & . KKI & SIDIL.R.F. &. $\mathrm{LV}$ & . R. & IR & \\
\hline & $\ldots M$ & $\ldots \ldots \ldots$ & P..S.I &. $\mathrm{GKN}$ & NITIL...F. & PLF & $\ldots Q$ & TR & \\
\hline & $\ldots M$ & $\ldots \ldots \ldots$ & L.F..L & . IT & $1 \ldots$ &.$L F$ & .R.Q & IR & \\
\hline & LL.S.T. . T & $\ldots Q$ & L...R.I. & SKKE & I.FYL.L..H & IS.M. .V.L. & $\ldots$ & & \\
\hline & .T...AS. .M & $\ldots \ldots$ & L.P. L..H. & $\ldots \mathrm{KI}$ & S.AIL...F. & AY.S..L.IP & $\ldots$ & JLL & \\
\hline ris & SL. . . . . . & $\ldots \ldots$ T. & L..S.S & $\ldots \mathrm{T}$ & $\ldots \ldots$ & .Y...YLV . & S... & FD & \\
\hline & $\ldots$. SF . . & L.QQ & L..T. & . KE. & NFF. & IF.L.FF.T. & .LT.F & JLL & \\
\hline ssum & LL.T. . & L. $\ldots$ & L...GT & L.NTPC & R.FL & SY... & IG. . F & I. . & \\
\hline \multirow[t]{2}{*}{ Psilotum } & YF...MI .L & $\ldots \ldots$ & L..V.I & $\ldots \mathrm{ET}$ & R. . I & FY...I & $\ldots$ & LS. & \\
\hline & 221 & 231 & 24 & 25 & & & $\left.\right|^{281}$ & & \\
\hline & KGTK & $\mathrm{KCY}$ & YFH & VSP & LTFI & $\mathrm{AK}$ & MINI & Is & \\
\hline & $\ldots$ & . SHI & D. & is & .Y.. & RIQ &. $\mathrm{LE}$ & & \\
\hline & E..H & $\mathrm{K}$. & $\mathrm{JE}$ & & VS.. & IE & TVTI & & S: \\
\hline & E..V & RS & H. . & & IS..S. & IE & TTTG & & \\
\hline & G. . F & ES & HC. & IG. &. $\mathrm{V}$ & RVG & ATEG & V. & \\
\hline & S..R & I.F.I & Q. .L &. $\mathrm{N}$ & ... & VG & T.GE & ¿L. & 50 \\
\hline & T. I & & $\ldots \mathrm{SI}$ & TSS & J.A.S... & . VL & .... & $\mathrm{v}$ & \\
\hline & & & HY. & & ..T.V & RVG & . AS & k. . & \\
\hline is & & & H. & & $\ldots \ldots$ & $\ldots$ &. EDE . . . CL & $\ldots$ & \\
\hline & E.FY & $F$ & FM. & QS & . $R$ & . RSI & SLDKS.TIYS & LLKTQ & \\
\hline ossum & E.SNY & . C & NH.S. & NS & IF ...T. & K. . & TMD. . & KV. & SL \\
\hline \multirow[t]{2}{*}{ Psilotum } & & & & & FS.M. . . F & HT. & . $\mathrm{HC}$ & & \\
\hline & 3 & 341 & 3 & 361 & 371 & 381 & I & i & \\
\hline & DDDI & & & IQY & KT & & RLFF & & 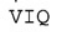 \\
\hline & .S. . & & & & & & $E Q$ & & I. \\
\hline & ..K. II & L.QV & ASM.Q & LIFL.QM. .D & S...G.R. & . LL & A. NQ & . RSS & S.R \\
\hline & ...L.R.VQ & . II & ASI . D. . RR & LR...L. . D & S...G.R. & $\ldots F L$ & LPKT & DKNQ.V.H.S & $L \cdot R$ \\
\hline & ...L.R.VQ & & .SI ..D. . RR & LK. . . . . D & N........ & T.SL & I.LD & SNNQ.V.H.S & \\
\hline & ...LKR.I. & & .SI .D. . RR & SKH. .... D & $\ldots \ldots \ldots$ & T.SL & VVSDN & SSNQ.V...N & IT \\
\hline & .S. .SKR.V. & L..I & .SRS.D. RK & LR..... D & $\ldots \ldots$ & $\ldots \mathrm{LI}$ & TFLKI & FSNQ . RD . & \\
\hline & ...L.R.LR & . RS. & .SID.D...R & LR. . . . . D & $\ldots \ldots$ & T.SI & .IL . .I & SNDR.I.C. . & \\
\hline & $\ldots$ LI & RSV & $. S I . N H \ldots F R$ & LR . F . . . . & $\ldots \ldots$ & T.I. & SFLRS & L..R.... & \\
\hline & .EE. LLN . K & $\ldots S F$ & . LIKKDI . R &. $\mathrm{K} \ldots \ldots$ & ...R.... & T.....IV & D.SLSSRR.K & ND.....ss. & \\
\hline & T.TT. & L...L. & .SSGLG . . R R & $R \ldots \ldots \ldots$ & $\ldots \ldots \ldots$ & .A. .RF . & . FNL. SVNPY & P.N. . C. . & $\mathrm{s}$ \\
\hline 51 & ..L.KYHQ & GDLS & S.D..WR & ...QL . . & $\ldots Q \ldots$ & T. . . RNHF.L & KFITTSKNPF & F.R.N.C. & \\
\hline
\end{tabular}

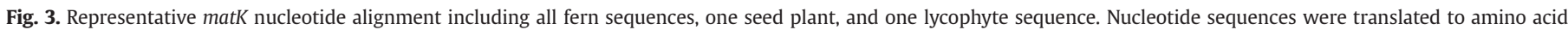

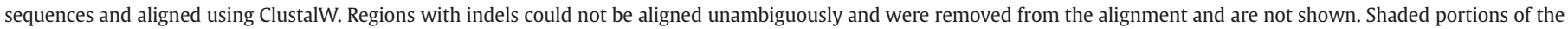
alignment represent the tether region including RT subdomains V, VI, and VII (amino acids 207-270) and domain X (amino acids 293-393).

We used the program package PAML (Yang, 1997) to evaluate models that allow ratios of nonsynonymous to synonymous substitution rates to vary among lineages in order to test whether levels of selective constraint vary among the lineages of ferns without the trnK intron, ferns with the trnK intron, and the rest of the tree. This method (Yang, 1998) requires a priori hypotheses of which branches on the tree vary, and models are compared using likelihood ratio tests. We constructed several models (Table 2) ranging from the simplest, with all 3 lineages assigned a single $d N /$ $\mathrm{d} S$ ratio (Model A), to the most general, with each of the three lineages assigned a separate ratio (Model D). PAML and another program package, HYPHY (Kosakovsky Pond et al., 2005), were both used to evaluate a free branch model, which assigns a separate ratio to each branch of the tree.

We also performed HYPHY GA Branch Analysis, using a genetic algorithm to assign $\mathrm{d} N / \mathrm{d} S$ ratios. Each branch on the phylogenetic tree was assigned to a $\mathrm{dN} / \mathrm{d} S$ ratio class with the optimal number of classes determined from the data (Kosakovsky Pond and Frost, 2005). We then looked for patterns in the way branches were assigned to ratio classes that might suggest differences between lineages.

\section{Results}

\subsection{Search for trnK intron in Adiantum}

Using the domain-by-domain folding method on the trnK intron/matK sequence regions of land plants, we recovered core secondary structural models consistent with well supported group II intron models (Michel et al., 1989) and identified several conserved sequence elements related to structures shared across taxa (Table 1). Most of these short (4-7 nucleotides) elements returned positive matches when used in localized sequence searches upstream and downstream of the matK ORF in A. capillus-veneris, but none was 
Table 2

Likelihood ratio tests of variation in $\mathrm{dN} / \mathrm{d} S$ ratios between the ferns with the trnK intron $(\mathrm{K}+$ ), the clade of ferns without the trnK intron ( $\mathrm{K}-$ ), and the rest of the tree (bg).

\begin{tabular}{|c|c|c|c|c|c|c|c|}
\hline $\mathrm{H}_{0}$ & Assumption & Models & $\mathrm{chi}^{2}$ & df & $P$ value & \multicolumn{2}{|c|}{ What are we asking? } \\
\hline $\mathrm{K}-=\mathrm{bg}$ & $\mathrm{K}+=\mathrm{bg}$ & $\mathrm{A}$ and $\mathrm{B}$ & 0.03 & 1 & 0.8543 & \multicolumn{2}{|c|}{ Does the K-minus clade differ from everything else? } \\
\hline $\mathrm{K}-=\mathrm{bg}$ & $\mathrm{K}+=$ free & C and D & 3.05 & 1 & 0.0807 & \multicolumn{2}{|c|}{ Does the K-minus clade differ from everything else if the K-plus ferns are free to vary? } \\
\hline root $=\mathrm{bg}$ & $\mathrm{K}+=\mathrm{bg}$ & $\mathrm{A}$ and $\mathrm{E}$ & 0.22 & 1 & 0.6423 & \multicolumn{2}{|c|}{ Does the single branch leading to the K-minus clade differ from everything else? } \\
\hline & & A and Free & 60.31 & 33 & $0.0026^{*}$ & \multicolumn{2}{|c|}{ Does a model with separate values for each branch fit the data better than a one ratio model? } \\
\hline Model A & & Model B & \multicolumn{2}{|c|}{ Model C } & \multicolumn{2}{|c|}{ Model D } & Model E \\
\hline$-\mathrm{K}$ & & $-\mathrm{K}-1$ & \multicolumn{2}{|c|}{$-\mathrm{K}-0$} & & $\mathrm{~K}-2$ & $1-K-0$ \\
\hline ᄂ $\mathrm{K}$ & & $-\mathrm{K}+0$ & & +1 & & +1 & $-\mathrm{K}+0$ \\
\hline$-b g$ & 0 & $-b g \quad 0$ & & $\lg 0$ & & g 0 & - bg 0 \\
\hline
\end{tabular}

$* P<0.01$.

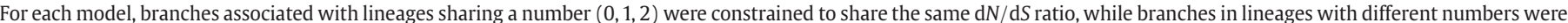
free to differ. For Model E, all branches were constrained to share a $\mathrm{dN} / \mathrm{d} S$ ratio except the single branch rooting the ferns without the trnK intron (root).

embedded in sequences that could be folded into RNA secondary structures resembling those of the trnK intron from which the element was derived. We found no evidence of potentially homologous helical structures and their associated conserved sequence elements that would indicate a trnK intron fragment. We conclude that matK is no longer associated with a divided, but functional, trnK intron in the chloroplast genome of A. capillusveneris.

BLAST searches using domain 5 sequences from each of six land plant taxa returned trnK intron sequences (but not other group II intron sequences) from nearly all the major plant groups including the fern, Angiopteris evecta. This is consistent with our expectation that the nucleotide sequence of domain 5 in the trnK intron is both unique to the trnK intron and highly conserved in land plant chloroplast genomes. These BLAST searches did not return a domain 5 sequence for the trnK intron in A. capillus-veneris. When these six domain 5 sequences were used for BLAST searches directly against the $A$. capillus-veneris chloroplast genome, only a few 11-nucleotide complements were returned with low expect values $(0.045$ to 1.9$)$, but none demonstrated a secondary structure that was potentially homologous to domain 5 of a group II intron. None of the searches identified a sequence in A. capillus-veneris that resembled the trnK intron in either its primary or secondary structure, strongly favoring the hypothesis that the trnK intron, or recognizable fragments thereof, is no longer present in this chloroplast genome.

\subsection{Phylogenetic analysis}

The matK consensus tree topology (Supplementary Fig. 2) is similar to published phylogenetic hypotheses with just a few differences, primarily on branches that are not strongly supported in analyses using other genes. Equisetum is placed as sister to the rest of the monilophytes, rather than sister to Angiopteris as hypothesized by Pryer et al. (2004) and Qiu et al. (2006) or sister to the core leptosporangiates (Marsilea, Dicksonia, Adiantum, and Pteridium) as hypothesized by Magallón and Sanderson (2005). Within the seed plants, relationships differ somewhat from published hypotheses (Hilu et al., 2003; Magallón and Sanderson, 2005; Qiu et al., 2006). Despite these differences, the relationships for the comparisons we want to make are consistent with published hypotheses - the ferns and the seed plants form monophyletic sister groups, and the ferns without the trnK intron (hereafter referred to as the "K-minus clade") form a well supported monophyletic group nested within ferns with the trnK intron (hereafter referred to as the "K-plus ferns"). The consensus tree topology could be biased by the small number of taxa included and also by the limitations of any single-gene phylogenetic analysis, so a constraint tree based on published topologies was used for subsequent analyses. Results of analyses using both topologies were compared to ensure that topology differences did not affect our inferences (data not shown).

\subsection{Tests of relaxed selective constraint}

The proportion of variable nucleotide alignment sites for K-plus ferns, the K-minus clade, and seed plants was lower than the percent total amino acid variation (K-plus ferns: $70 \%$ vs. $81 \%$; K-minus clade: $67 \%$ vs. $82 \%$; seed plants: $58 \%$ vs. $74 \%$ ). This is consistent with values reported for other plant groups (Hilu and Liang, 1997) and suggests a lack of strong functional constraint on the gene as a whole since nucleotide variation typically translates into lower amino acid variation in functionally constrained genes (Graur and Li, 2000). However, any direct comparison of the percentage variation between $\mathrm{K}$-plus ferns, the K-minus clade, and seed plants is probably inappropriate as these values are likely biased by the different sample sizes used and the phylogenetic relationships among the groups.

Whereas the percentage variation and absolute number of variable nucleotides and amino acids may be biased, the pattern of variation or distribution of relatively conserved or variable regions along the sequence can be compared directly. The pattern of nucleotide and amino acid variation along the length of the matK sequence is similar in K-plus ferns, the K-minus clade, and seed plants (Fig. 4), and is similar to the pattern described by Hilu et al. (2003) in the matK sequence for a large sample of angiosperms. The three groups have highly variable sequences but a region with less variability in one group tends to have less variability in the other groups as well. All three groups have regions of relatively conserved sequence located approximately 300 nucleotides ( 100 amino acids) from the $5^{\prime}$ end of our alignment, and approximately 200 nucleotides ( 65 amino acids) from the $3^{\prime}$ end of our alignment, with the latter region corresponding to part of domain X. These same low variability regions have been identified in other plant groups as well, including the nonphotosynthetic, trnK-lacking Epifagus (Hilu and Liang, 1997; Young and dePamphilis, 2000). All three groups also show a slight reduction in sequence variability within the tether region, but it is less conserved than the other two regions.

Likelihood ratio tests using PAML did not find significant differences between models varying in the levels of heterogeneity in the $\mathrm{dN} / \mathrm{d} S$ ratio among lineages (Table 2, Table 3 ). The model with one ratio for the K-minus clade and one for the rest of the tree (Model B) does not fit the data significantly better than a single ratio model (Model A), suggesting that the $\mathrm{dN} / \mathrm{d} S$ ratio for the K-minus clade is not different from the rest of the tree. This is the case even if the $\mathrm{d} N / \mathrm{d} S$ ratio for the K-plus ferns is allowed to vary (Model C vs. Model D). To allow for the possibility that purifying selection on matK was 


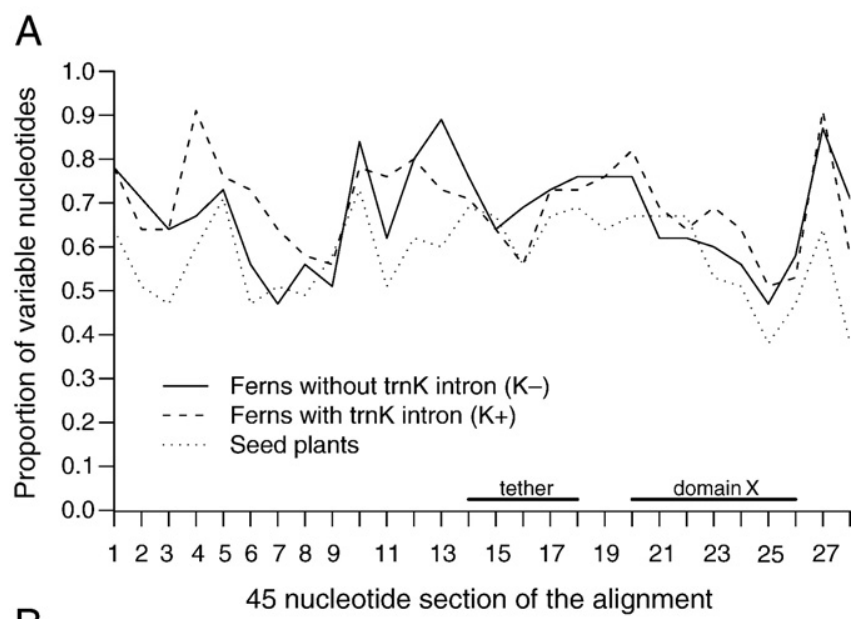

B

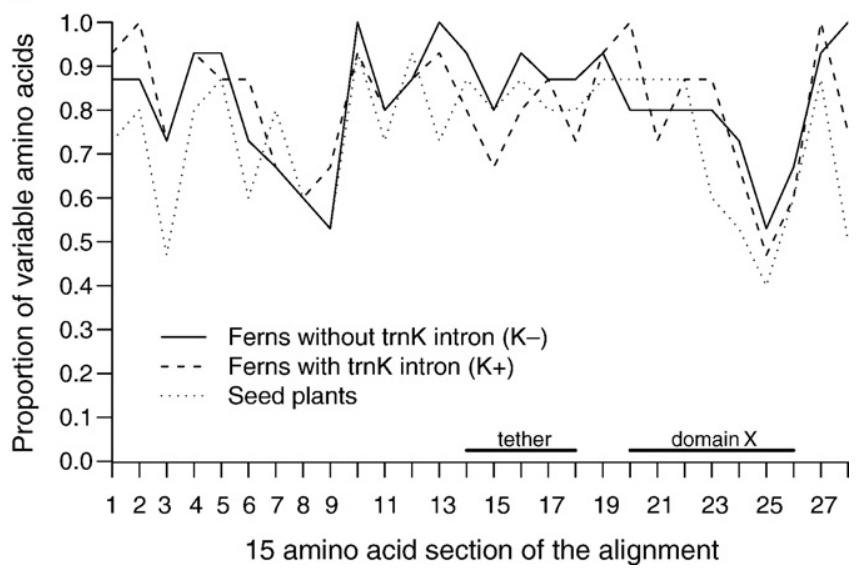

Fig. 4. Comparison of patterns of distribution of variable alignment positions for ferns without the trnK intron $(\mathrm{K}-)$, ferns with the trnK intron $(\mathrm{K}+)$, and seed plants. (A) Proportion of variable positions in each 45 nucleotide section of the matK nucleotide alignment. Each number on the $x$-axis represents a 45 nucleotide section of the alignment. (B) Proportion of variable positions in each 15 amino acid section of the matK amino acid alignment. Each number on the $x$-axis represents a 15 amino acid section of the alignment. Regions containing indels are excluded from the alignment. The approximate position of the tether region and domain $\mathrm{X}$ are labeled on the $x$-axis.

diminished following the inversion and loss of the intron but then increased again, we tested specifically for a change in $\mathrm{d} N / \mathrm{d} S$ on the branch where the inversion that separated matK from trnK and its intron occurred (Model E). We tested whether a model with one ratio for that single branch and another ratio for the rest of the tree fits the data better than the one ratio model (Model E vs. Model A), but found no significant difference.

Table 3

Estimates of $\mathrm{dN} / \mathrm{d} S$ ratios and log likelihood values for the ferns with the trnK intron $(\mathrm{K}+)$, ferns without the trnK intron $(\mathrm{K}-)$, and the rest of the tree $(\mathrm{bg})$, under different models used in branch model tests.

\begin{tabular}{llll}
\hline Model & $\mathrm{d} N / \mathrm{d} S$ & $\ln$ (Likelihood) & Parameters \\
\hline $\mathrm{A}$ & $\mathrm{bg}=\mathrm{K}+=\mathrm{K}-=0.2899$ & -18539.203 & 36 \\
$\mathrm{~B}$ & $\mathrm{bg}=\mathrm{K}+=0.2913$ & -18539.186 & 37 \\
& $\mathrm{~K}-=0.2866$ & -18531.462 & 37 \\
$\mathrm{C}$ & $\mathrm{bg}=\mathrm{K}-=0.3188$ & & 38 \\
& $\mathrm{~K}+=0.2187$ & -18529.936 & \\
$\mathrm{D}$ & $\mathrm{bg}=0.3399$ & & 37 \\
& $\mathrm{~K}+=0.2185$ & \\
$\mathrm{E}$ & $\mathrm{K}-=0.2878$ & -18539.095 & \\
\hline
\end{tabular}

For Model E the single branch rooting the clade of ferns without the trnK intron (root) is compared to the rest of the tree.
We used a free ratio model to infer a separate $\mathrm{d} N / \mathrm{d} S$ ratio for each branch of the tree (Fig. 5). The free model analysis was performed using both PAML and HYPHY, and though the two software packages gave slightly different values for $\mathrm{d} N$ and $\mathrm{d} S$, the ratios were identical. Likelihood ratio tests show that the free ratio model fits the data significantly better than the one ratio model (Model A) (Table 2, Table 3 ). The $\mathrm{d} N / \mathrm{d} S$ ratios for nearly all branches were less than one, suggesting mild purifying selection on the gene as a whole, consistent with tests of selection on matK in other taxa using protein side-chain composition (Barthet and Hilu, 2008). There is no obvious increase in $\mathrm{d} N / \mathrm{d} S$ ratios on the branches associated with the K-minus clade. In general, values for the branches associated with the K-minus clade fall between those for the K-plus fern lineages and those for the seed plant lineages. There are three branches in the tree with $\mathrm{dN} / \mathrm{d} S$ values greater than one, but these all have very short branch lengths so it is not clear whether the high ratios are valid or are due to chance. However, these large or infinite $\mathrm{d} N / \mathrm{d} S$ values are not assigned to branches associated with the K-minus clade. These results do not suggest that the matK sequence of the K-minus clade is under relaxed selective constraint or positive selection relative to the other groups. If anything, the K-minus clade is under slightly stronger purifying selection than the seed plants and slightly weaker purifying selection than the K-minus ferns.

The HYPHY Genetic Algorithm Branch Analysis assigned each branch to one of four classes ranging from $\mathrm{dN} / \mathrm{d} S=0.139$ to 0.531 (Fig. 6). None of the branches associated with the K-minus clade was assigned to the highest $\mathrm{d} N / \mathrm{d} S$ class. All but one of these branches were assigned to the 2nd highest (and most common) class. Within all the ferns, the only two branches assigned to the highest $\mathrm{d} N / \mathrm{d} S$ class were the branch rooting Angiopteris, Equisetum, and the Leptosporangiate ferns, and the branch leading to Ophioglossum. The rest of the

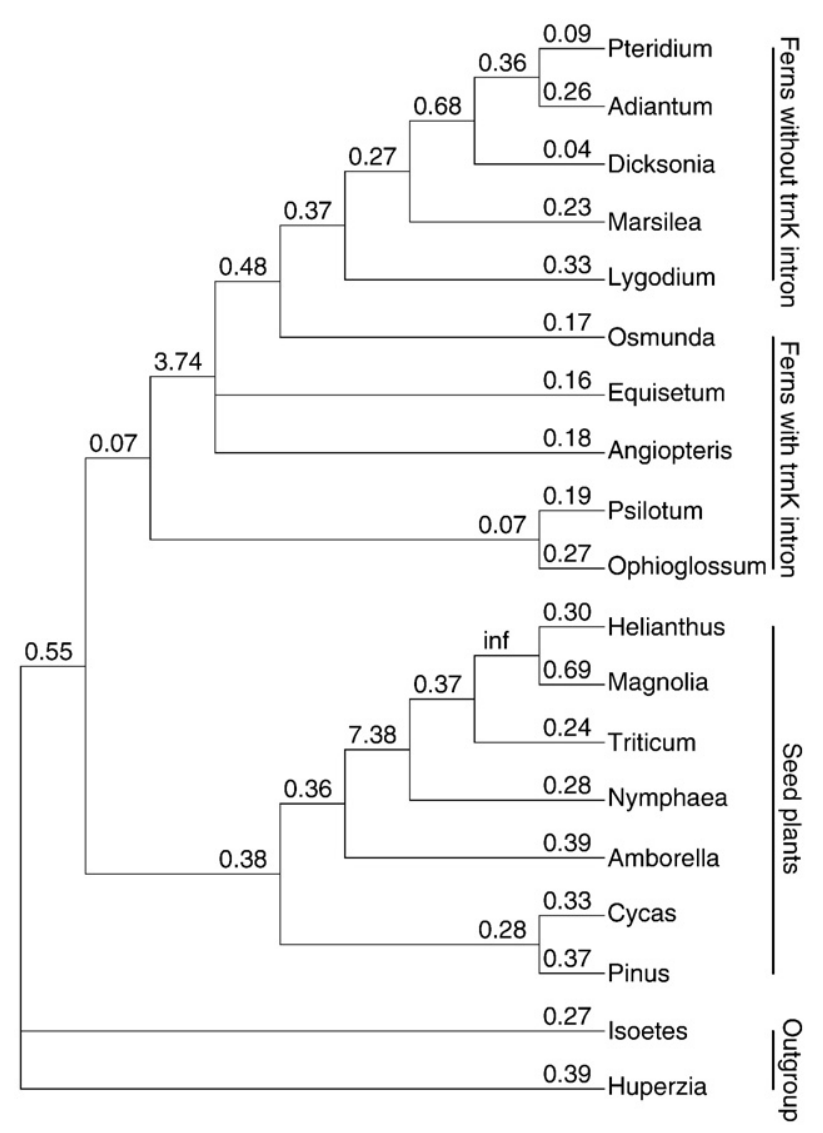

Fig. 5. Constraint tree with Free Model $\mathrm{d} N / \mathrm{d} S$ ratio values with a separate value assigned to each branch. Tree topology is constrained to be consistent with published seed plant and monilophyte phylogenies. 


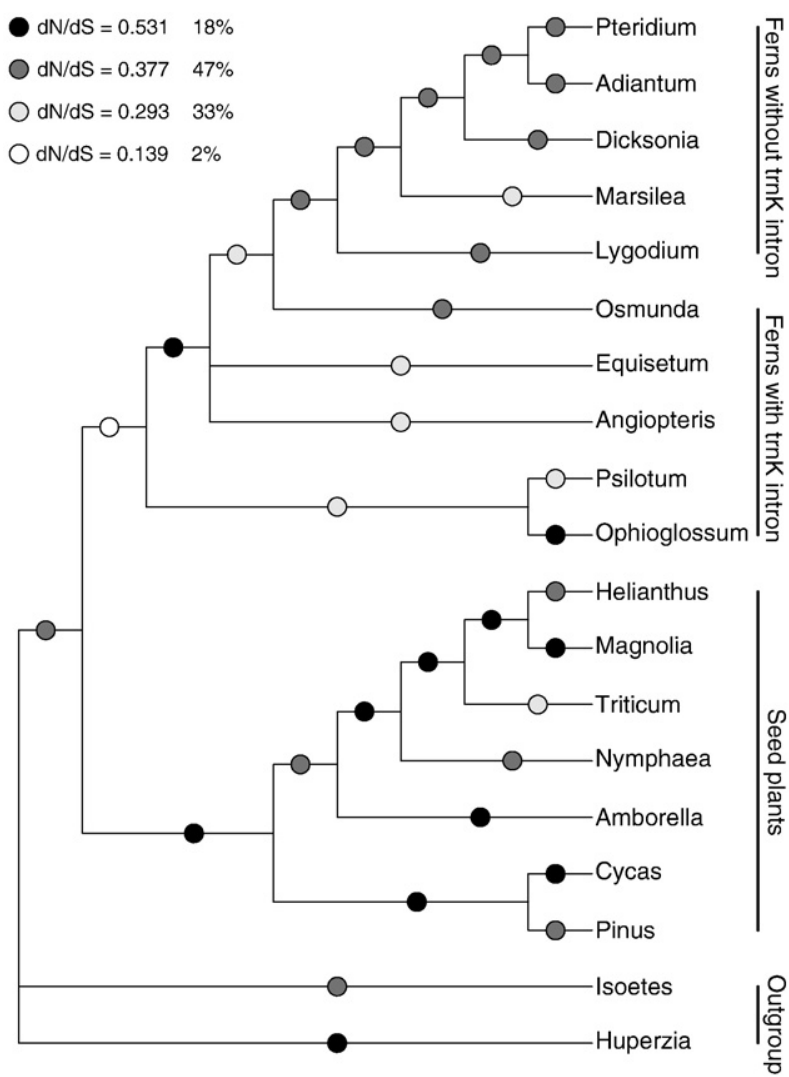

Fig. 6. HYPHY Genetic Algorithm Branch Analysis tree for the full matK sequence. Branches are assigned to $\mathrm{d} N / \mathrm{d} S$ ratio categories with the optimal number of categories determined from the data. The percentages for the branch classes represent the proportion of the tree length (expected substitutions per site per unit time) evolving under that value of $\mathrm{dN} / \mathrm{d} S$. Tree topology is constrained to be consistent with published seed plant and monilophyte phylogenies.

branches allocated to the highest class were in the seed plants and outgroup. The only branch included in the lowest $\mathrm{dN} / \mathrm{d} S$ class is the branch rooting the ferns. Overall, the class assignments mirror the results of the likelihood ratio tests, with the fern branches generally in the same or lower $\mathrm{dN} / \mathrm{d} S$ classes than the seed plants and outgroup. Within the ferns, the branches for the K-minus clade tend to belong to higher classes than the K-plus fern branches, but still lower than those for the rest of the tree. None of the results suggests that there is relaxed purifying selection in the lineages where matK has been separated from trnK and its intron.

\section{Discussion}

Our investigation of the atypical trnK/matK condition in the $A$. capillus-veneris chloroplast genome (which is shared by other ferns in the K-minus clade) leads us to conclude that the trnK intron has been lost even though its IEP, matK, is retained. The position of the matK ORF at the border of an inferred inversion event had allowed for the possibility that the trnK intron was still functional in the genome as a trans-spliced, divided intron. If so, matK would be flanked by one or more of the trnK intron domains, and the highly conserved, diagnostic domain 5 sequence would be detectable in the genome. However, we found no evidence for any conserved trnK intron secondary structure or sequence motifs flanking the matK ORF, or for conserved trnK domain 5 sequence elements in the complete $A$. capillus-veneris chloroplast genome. These observations favor the hypothesis that the trnK intron was disrupted by an inversion event in an ancestor of the higher ferns, failed to persist by trans-splicing mechanisms in the chloroplast genome, and was subsequently degraded and lost.
If matK was functioning primarily as a splice factor for trnK and its intron, once trnK was lost, we would expect matK to be released from this particular selective constraint and to either accumulate substitutions and become degraded, or optimize to its role as a generalist catalyst of splicing IIA introns, or less likely, to gain new function. However, the similar distributions of conserved nucleotides and amino acids in the matK sequences of seed plants, K-plus ferns, and the K-minus clade suggest that substitutions are not being accumulated differently in the matKs that exist without the trnK intron. Additionally, analyses of $\mathrm{dN} / \mathrm{d} S$ ratios using several methods including the entire coding sequence as well as specifically focusing on the functional domains - do not support the hypothesis that matK is under relaxed selective constraint in taxa without trnK and its intron, as would be expected if the gene was no longer functional in these taxa. If anything, the $\mathrm{dN} / \mathrm{d} S$ analyses suggest that matK in the ferns without the trnK intron has been under a similar level of selective constraint to that of the seed plants, whereas in the ferns with the trnK intron, matK is slightly more constrained.

The presence of matK cDNAs in A. capillus-veneris (Wolf et al., 2004) and another fern in the K-minus clade (Barthet and Hilu, 2007) suggests that matK continues to function despite the absence of the trnK intron. If matK were performing a new role in the genomes of ferns without the trnK intron, we would expect to see evidence of positive selection as the sequence responded to different selective forces, but there is no evidence of positive selection in any of the analyses. So, even after the loss of trnK and its intron, matK is apparently performing a largely similar function, consistent with the hypothesis that matK is involved in splicing other group IIA introns in the chloroplast (Vogel et al., 1999). Other genes in the A. capillusveneris chloroplast genome with group II introns are atpF, clpP, rpl2, rps12, trnA, trnI, and trnV (Wolf et al., 2003; Funk et al., 2007).

The hypothesized inversion (Hasebe and Iwatsuki, 1992; Stein et al., 1992; Roper, 2007) that appears to have disrupted the intron is shared by members of a clade that diverged approximately 265 mya (Pryer et al., 2004) and includes nearly 90\% of all fern species. This indicates that the matK condition in these taxa is both ancient and stable and contrasts with the other examples of inferred maturase isolation - the reduced chloroplast genomes of E. virginiana (Wolfe et al., 1992; Ems et al., 1995) and C. reflexa (Funk et al., 2007), which are members of much younger angiosperm clades.

Because the genomes of nearly $90 \%$ of all extant fern species do not have the typical trnK/matK arrangement, and many of the primers used to amplify matK in other plant groups are within trnK or its intron, ferns have been underrepresented in previous analyses of matK. The high rate of nucleotide substitution in matK makes it difficult to design primers that are conserved across large taxonomic groups, and taxon-specific primers are often required (Hilu et al., 2003). A recent proposal of two options for three-gene land plant DNA barcodes included matK in both options, but noted that additional work was needed to improve the performance of the primer sets (Chase et al., 2007). We have so far been unable to design a single set of primers to amplify matK across all the fern taxa used in this study, but designed two sets: one in sequence conserved across the early diverging fern lineages and one in sequence conserved across the more recently derived lineages within the K-minus clade (Fig. 2). There is some overlap in the taxa that each set will amplify, but so far the "early" fern primers have been tested successfully on Osmunda, Lygodium, Gleichenia, Marsilea, and Dicksonia and the "modern" fern primers have been tested successfully on Adiantum, Dicksonia, and Pteridium. Because these primers are located within the matK sequence they cannot amplify the entire matK gene. But, by combining these primers with knowledge of the gene order surrounding matK in ferns with and without trnK (Fig. 1), the entire matK sequence may be amplified using published chlB or rps16 primers for the upstream flank and $n d h B$ (K-minus clade) or psbA (K-plus ferns) for the downstream flank (Roper, 2007). The primers designed for this study should allow matK to be amplified and sequenced for most fern taxa. 


\subsection{Evolutionary significance of maturase isolation}

Close functional association of introns and their encoded maturase is common among prokaryotic, protist, and fungal group II introns (Lambowitz and Perlman, 1990; Moran et al., 1995; Saldanha et al., 1999; Huang et al., 2005), many of which appear to have co-evolved with their IEPs (Costa et al., 1997; Toor et al., 2001; Zimmerly et al., 2001). Liere and Link (1995) established that matK binds preferentially to trnK intron pre-mRNAs in the mustard Sinapis alba when given the opportunity. Although matK is typical in that it maintains a tight functional association with its encoding intron, it is also unusual in its apparent contribution to splicing reactions of other group IIA introns (Vogel et al., 1999). One plausible history for the advent of matK's generalist nature is given here. It is thought that mobile group II introns invaded ancestral chloroplast genomes sometime prior to the divergence of the charophytes and embryophytes (Toor et al., 2001; Sanders et al., 2003). As most extant mobile group II introns use IEPs for retrotranspositioning (Zimmerly et al., 2001), it is likely that each invading intron carried a maturase ORF in its domain 4 and one of these was the ancestor of matK. Consistent with this hypothesis are the observations that matK is always associated with the trnK intron when both are present, and that chloroplast genomes that lack group II introns also lack matK (Turmel et al., 1999; Lemieux et al., 2000).

After their establishment in plastid genomes, most chloroplast group II introns seem to have shifted from a specific interaction with their IEPs to a more relaxed association with a variety of spliceassisting proteins such as those encoded by nuclear genes crs1 and crs2 (Jenkins et al., 1997), plastid ribosomes (Hess et al., 1994), and other as yet to be determined factors (Vogel et al., 1999; Matsuura et al., 2001; Toor et al., 2001). Such associations would have lessened the constraints on chloroplast IEPs, eventually leading to the degradation of ORFs in nearly all group II introns. Although there are at least four protein-assisted pathways for splicing different assemblages of introns in plant chloroplasts, introns belonging to subclass IIA may continue to rely on matK (Jenkins et al., 1997; Vogel et al., 1999). The presence of matK in the reduced chloroplast genomes of $E$. virginiana and $C$. reflexa, and our example of its preservation after trnK intron loss in higher ferns, seem to support this model.

To our knowledge, the higher ferns represent only the third reported case of intron loss with IEP retention - and the first where the intron loss was attributable to an inversion. The expected co-dependence of a group II intron and its IEP makes such an event remarkable. However, plant chloroplast genomes offer an unusual opportunity for IEP isolation due to the seeming dependence of multiple group II introns on a single splice-assisting maturase. The apparent conservation of selection on protein sites suggests that matK was performing this generalist function even before this ancient inversion led to the loss of trnK and its intron in a fern ancestor. Our study presents a plausible example of a molecule (maturase K) shifting from its primary function as a specific splice factor for the trnK intron to a generalist function as a splice factor for multiple group II introns, and finally to being maintained in its new capacity in the plastid genome despite the loss of its co-evolved and previously dependent molecule, the trnK intron.

\section{Acknowledgements}

Thanks to Dawn Simon, Josh Der, Mark Ellis, and anonymous reviewers for their comments on an earlier draft of the manuscript. This research was funded by National Science Foundation grant DEB0228432 to PGW, and a Utah State University Vice President of Research fellowship to AMD.

\section{Appendix A. Supplementary data}

Supplementary data associated with this article can be found, in the online version, at doi:10.1016/j.gene.2009.02.006.

\section{References}

Barthet, M.M., Hilu, K.W., 2007. Expression of matK: functional and evolutionary implications. Am. J. Bot. 94, 1402-1412.

Barthet, M.M., Hilu, K.W., 2008. Evaluating evolutionary constraint on the rapidly evolving gene matK using protein composition. J. Mol. Evol. 66, 85-97.

Bonen, L., 1993. Trans-splicing of pre-mRNA in plants, animals, and protists. FASEB J. 7, 40-46.

Chapdelaine, Y., Bonen, L., 1991. The wheat mitochondrial gene for subunit I of the nadH dehydrogenase complex - a trans-splicing model for this gene-in-pieces. Cell 65, 465-472.

Chase, M.W., Cowan, R.S., Hollingsworth, P.M., et al., (19 co-authors), 2007. A proposal for a standardised protocol to barcode all land plants. Taxon 56, 295-299.

Costa, M., Fontaine, J.-M., Loiseaux-de Goër, S., Michel, F., 1997. A group II self-splicing intron from the brown alga Pylaiella littoralis is active at unusually low magnesium concentrations and forms populations of molecules with a uniform conformation. J. Mol. Biol. 274, 353-364.

Doyle, J.J., Doyle, J.L., 1987. A rapid DNA isolation procedure for small quantities of fresh leaf tissue. Phytochem. Bull. 19, 11-15.

Ems, S.C., Morden, C.W., Dixon, C.K., Wolfe, K.H., dePamphilis, C.W., Palmer, J.D., 1995 Transcription, splicing and editing of plastid RNAs in the nonphotosynthetic plant Epifagus virginiana. Plant Mol. Biol. 29, 721-733.

Funk, H.T., Berg, S., Krupinska, K., Maier, U.G., Krause, K., 2007. Complete DNA sequences of the plastid genomes of two parasitic flowering plant species, Cuscuta reflexa and Cuscuta gronovii. BMC Plant Biol. 7, 45.

Graur, D., Li, W.-H., 2000. Fundamentals of Molecular Evolution, Second ed. Sinauer Associates, Sunderland, Massachusetts.

Hasebe, M., Iwatsuki, K., 1992. Gene localization on the chloroplast DNA of the maiden hair fern; Adiantum capillus-veneris. Bot. Mag. Tokyo 105, 413-419.

Hausner, G., Olson, R., Simon, D., Johnson, I., Sanders, E.R., Karol, K.G., McCourt, R.M. Zimmerly, S., 2006. Origin and evolution of the chloroplast trnK (matK) intron: a model for evolution of group II intron RNA structures. Mol. Biol. Evol. 23, 380-391.

Hess, W.R., Hoch, B., Zeltz, P., Hübschmann, T., Kössel, H., Börner, T., 1994. Inefficient rpl2 splicing in barley mutants with ribosome-deficient plastids. Plant Cell 6, 1455-1465.

Hilu, K.W., Borsch, T., Müller, K., et al., (16 co-authors), 2003. Angiosperm phylogeny based on matK sequence information. Am. J. Bot. 90, 1758-1776.

Hilu, K.W., Liang, H., 1997. The matK gene: sequence variation and application in plant systematics. Am. J. Bot. 84, 830-839.

Huang, H.-R., Rowe, C.E., Mohr, S., Jiang, Y., Lambowitz, A.M., Perlman, P.S., 2005. The splicing of yeast mitochondrial group I and group II introns requires a DEAD-box protein with RNA chaperone function. Proc. Natl. Acad. Sci. U. S. A. 102, 163-168.

Huelsenbeck, J.P., Ronquist, F., 2001. MRBAYES: Bayesian inference of phylogenetic trees. Bioinformatics 17, 754-755.

Jarrell, K.A., Dietrich, R.C., Perlman, P.S., 1988. Group II intron domain 5 facilitates a trans-splicing reaction. Mol. Cell. Biol. 8, 2361-2366.

Jenkins, B.D., Kulhanek, D.J., Barkan, A., 1997. Nuclear mutations that block group II RNA splicing in maize chloroplasts reveal several intron classes with distinct requirements for splicing factors. Plant Cell 9, 283-296.

Kelchner, S.A., 2000. The evolution of non-coding chloroplast DNA and its application in plant systematics. Ann. Mo. Bot. Gard. 87, 482-498.

Kelchner, S.A., 2002. Group II introns as phylogenetic tools: structure, function, and evolutionary constraints. Am. J. Bot. 89, 1651-1669.

Knoop, V., Altwasser, M., Brennicke, A., 1997. A tripartite group II intron in mitochondria of an angiosperm plant. Mol. Gen. Genet. 255, 269-276.

Kosakovsky Pond, S.L., Frost, S.D.W., 2005. A genetic algorithm approach to detecting lineage-specific variation in selection pressure. Mol. Biol. Evol. 22, 478-485.

Kosakovsky Pond, S.L., Frost, S.D.W., Muse, S.V., 2005. HyPhy: hypothesis testing using phylogenies. Bioinformatics 21, 676-679.

Lambowitz, A.M., Perlman, P.S., 1990. Involvement of aminoacyl-transfer RNAsynthetases and other proteins in group-I and group-II intron splicing. Trends Biochem. Sci. 15, 440-444.

Lemieux, C., Otis, C., Turmel, M., 2000. Ancestral chloroplast genome in Mesostigma viride reveals an early branch of green plant evolution. Nature 403, 649-652.

Liere, K., Link, G., 1995. RNA-binding activity of the matK protein encoded by the chloroplast trnK intron from mustard (Sinapis alba L). Nucleic Acids Res. 23, 917-921.

Magallón, S.A., Sanderson, M.J., 2005. Angiosperm divergence times: the effect of genes, codon positions, and time constraints. Evolution 59, 1653-1670.

Malek, O., Knoop, V., 1998. Trans-splicing group II introns in plant mitochondria: the complete set of cis-arranged homologs in ferns, fern allies, and a hornwort. RNA 4, 1599-1609.

Matsuura, M., Noah, J.W., Lambowitz, A.M., 2001. Mechanism of maturase-promoted group II intron splicing. EMBO J. 20, 7259-7270.

Michel, F., Umesono, K., Ozeki, H., 1989. Comparative and functional anatomy of group II catalytic introns - a review. Gene 82, 5-30.

Mohr, G., Perlman, P.S., Lambowitz, A.M., 1993. Evolutionary relationships among group II intron-encoded proteins and identification of a conserved domain that may be related to maturase function. Nucleic Acids Res. 21, 4991-4997.

Moran, J.V., Zimmerly, S., Eskes, R., Kennell, J.C., Lambowitz, A.M., Butow, R.A., Perlman, P.S., 1995. Mobile group II introns of yeast mitochondrial DNA are novel site-specific retroelements. Mol. Cell. Biol. 15, 2828-2838.

Nylander, J.A.A., 2004. MrModeltest v2. Program distributed by the author. Evolutionary Biology Center, Uppsala University.

Posada, D., Crandall, K.A., 1998. MODELTEST: testing the model of DNA substitution. Bioinformatics. 14, 817-818.

Pryer, K.M., Schuettpelz, E., Wolf, P.G., Schneider, H., Smith, A.R., Cranfill, R., 2004. Phylogeny and evolution of ferns (monilophytes) with a focus on the early leptosporangiate divergences. Am. J. Bot. 91, 1582-1598. 
Qiu, Y.-L., Palmer, J.D., 2004. Many independent origins of trans splicing of a plant mitochondrial group II intron. J. Mol. Evol. 59, 80-89.

Qiu, Y.-L., Libo, L., Wang, B., et al. (21 co-authors), 2006. The deepest divergences in land plants inferred from phylogenomic evidence. 103, 15511-15516.

Ronquist, F., Huelsenbeck, J.P., 2003. MrBayes 3: Bayesian phylogenetic inference under mixed models. Bioinformatics 19, 1572-1574.

Roper, J.M., 2007. Structural Evolution of Fern Chloroplast Genomes. Master of Science thesis. Utah State University, Logan, Utah, USA.

Saldanha, R., Chen, B., Wank, H., Matsuura, M., Edwards, J., Lambowitz, A.M., 1999. RNA and protein catalysis in group II intron splicing and mobility reactions using purified components. Biochemistry 38, 9069-9083.

Sanders, E.R., Karol, K.G., McCourt, R.M., 2003. Occurrence of matK in a trnK group II intron in charophyte green algae and phylogeny of the Characeae. Am. J. Bot. 90, 628-633.

Stein, D.B. Conant, D.S, Ahearn, M.E., Jordan, ET., Kirch, S.A., Hasebe, M. Iwatsuki, K., Tan, M. K., Thomson, J.A., 1992. Structural rearrangements of the chloroplast genome provide an important phylogenetic link in ferns. Proc. Natl. Acad. Sci. U. S. A. 89, 1856-1860.

Toor, N., Hausner, G., Zimmerly, S., 2001. Coevolution of group II intron RNA structures with their intron-encoded reverse transcriptases. RNA 7, 1142-1152.

Turmel, M., Otis, C., Lemieux, C., 1999. The complete chloroplast DNA sequence of the green alga Nephroselmis olivacea: insights into the architecture of ancestral chloroplast genomes. Proc. Natl. Acad. Sci. U. S. A. 96, 10248-10253.

Vogel, J., Hübschmann, T., Börner, T., Hess, W.R., 1997. Splicing and intron-internal RNA editing of trnK-matK transcripts in barley plastids: support for matK as an essential splice factor. J. Mol. Biol. 270, 179-187.
Vogel, J., Börner, T., Hess, W.R., 1999. Comparative analysis of splicing of the complete set of chloroplast group II introns in three higher plant mutants. Nucleic Acids Res. 27, 3866-3874.

Wolf, P.G., Rowe, C.A., Sinclair, R.B., Hasebe, M., 2003. Complete nucleotide sequence of the chloroplast genome from a leptosporangiate fern, Adiantum capillus-veneris L. DNA Res. 10, 59-65.

Wolf, P.G., Rowe, C.A., Hasebe, M., 2004. High levels of RNA editing in a vascular plant chloroplast genome: analysis of transcripts from the fern Adiantum capillus-veneris. Gene 339, 89-97.

Wolfe, K.H., Morden, C.W., Palmer, J.D., 1992. Function and evolution of a minimal plastid genome from a nonphotosynthetic parasitic plant. Proc. Natl. Acad. Sci. U. S. A. 89, 10648-10652.

Yang, Z., 1997. PAML: a program package for phylogenetic analysis by maximum likelihood. Comput. Appl. Biosci. 13, 555-556.

Yang, Z., 1998. Likelihood ratio tests for detecting positive selection and application to primate lysozyme evolution. Mol. Biol. Evol. 15, 568-573.

Yang, Z., Bielawski, J.P., 2000. Statistical methods for detecting molecular adaptation. Trends Ecol. Evol. 15, 496-503.

Young, N.D., dePamphilis, C.W., 2000. Purifying selection detected in the plastid gene matK and flanking ribozyme regions within a group II intron of nonphotosynthetic plants. Mol. Biol. Evol. 17, 1933-1941.

Zimmerly, S., Hausner, G., Wu, X.C., 2001. Phylogenetic relationships among group II intron ORFs. Nucleic Acids Res. 29, 1238-1250.

Zuker, M., 2003. Mfold web server for nucleic acid folding and hybridization prediction. Nucleic Acids Res. 31, 3406-3415. 